Comment. Math. Helv. 78 (2003) 463-485

0010-2571/03/030463-23

DOI $10.1007 / \mathrm{s} 00014-003-0767-8$
(C) 2003 Birkhäuser Verlag, Basel

Commentarii Mathematici Helvetici

\title{
Dynamical properties of the space of Lorentzian metrics
}

Pierre Mounoud

À la mémoire de mon père

\begin{abstract}
We study the mechanisms of the non properness of the action of the group of diffeomorphisms on the space of Lorentzian metrics of a compact manifold. In particular, we prove that nonproperness entails the presence of lightlike geodesic foliations of codimension 1 . On the 2-torus, we prove that a metric with constant curvature along one of its lightlike foliation is actually flat. This allows us to show that the restriction of the action to the set of non-flat metrics is proper and that on the set of flat metrics of volume 1 the action is ergodic. Finally, we show that, contrarily to the Riemannian case, the space of metrics without isometries is not always open.
\end{abstract}

Mathematics Subject Classification (2000). 58D17 (53C50, 53C12).

Keywords. Space of Lorentzian metrics, lightlike geodesic foliation, approximately stable vector, metrics without isometries.

\section{Introduction}

We study the action of the group of diffeomorphisms of a compact manifold $M$, denoted by $\operatorname{Diff}(M)$, on the space of Lorentzian metrics of $M$, denoted by $\mathcal{L}(M)$. An important motivation is to understand the space of Lorentzian structures, i.e. the quotient space. In the Riemannian case this action is well understood, thanks to the work of D. G. Ebin [Eb]. He proved in particular that it is proper.

Even though properness has no reason to hold in the pseudo-Riemannian case, the situation is rather intricate. On one hand we can single out manifolds for which this action is indeed proper. On the other hand non properness turns out to be a very special property. This is related to the works of R. Zimmer [Zi], G. D'Ambra and M. Gromov [D-Gr], S. Adams and G. Stuck [A-S], or A. Zeghib [Ze1] and [Ze2], about non compact isometry groups of closed Lorentzian manifolds.

Our first result is a generalization of the main theorem of the article [Ze4] by A. Zeghib which deals with non-equicontinuous sequences of isometries of a compact Lorentzian manifold. This result enables us to know how the properness fails. 
As we do not want to be too technical in this introduction, we formulate a weak version of theorem 2.2 as follows.

Theorem. Let $K$ and $L$ be two compact subsets of $\mathcal{L}(M)$ (for the $C^{2}$-topology) such that $\left\{\varphi \in \operatorname{Diff}(M) \mid \varphi^{*} K \cap L \neq \emptyset\right\}$ is not compact. Then both $K$ and $L$ contain metrics which possess a Lipschitz, codimension 1 , totally geodesic and lightlike foliation.

Recall that a submanifold of $(M, h)$ is lightlike if it is everywhere tangent to the light cone, i.e. if the restriction of the metric is everywhere degenerate. As in Zeghib's theorem, the foliation has a dynamical meaning: its tangent is the set of "approximately stable vectors" relative to a given sequence of diffeomorphisms. When $K$ and $L$ are reduced to a point, this theorem describes metrics with non compact isometry group, it is Zeghib's theorem.

The third section of this article is devoted to compact Lorentzian surfaces, i.e. to the torus and the Klein bottle (since the Euler characteristic must vanish). In this case we are able to be more precise in the description of the non-properness. It comes from the fact that the metrics described by the previous theorem have constant curvature along one of their lightlike foliations. Studying those metrics we obtain the following global geometric property of Lorentzian tori.

Theorem 3.1. A Lorentzian metric on the torus $\mathbf{T}^{2}$ whose curvature is constant along one of its lightlike foliations is flat.

Hence, on the torus the non-properness is localized on flat metrics. We still have to understand the restriction of the action to the set of flat metrics, that we will denote by $\mathcal{F}\left(\mathbf{T}^{2}\right)$. The volume functional is clearly invariant under the action of $\operatorname{Diff}\left(\mathbf{T}^{2}\right)$. It is therefore natural to restrict the action to the set of flat metrics of volume 1 , that we denote by $\mathcal{F}_{1}\left(\mathbf{T}^{2}\right)$. Next, in order to obtain a finite dimensional problem, we take the quotient of this set by the proper action of $\operatorname{Diff}_{0}\left(\mathbf{T}^{2}\right)$, the group of diffeomorphisms isotopic to the identity. The quotient space is diffeomorphic to the set of quadratic forms of signature $(1,1)$ and of determinant 1 , i.e. to $S L(2, \mathbb{R}) / S O(1,1)$. Furthermore, it is well known that $\operatorname{Diff}^{+}\left(\mathbf{T}^{2}\right) / \operatorname{Diff}_{0}\left(\mathbf{T}^{2}\right)$ is isomorphic to $S L(2, \mathbb{Z})$. Therefore the problem is now to understand the action of $S L(2, \mathbb{Z})$ on $S L(2, \mathbb{R}) / S O(1,1)$. This action being ergodic, we can state the following (cf. corollary 3.4 and theorem 3.8 ).

Theorem. 1. The action of $\operatorname{Diff}\left(\mathbf{T}^{2}\right)$ on $\mathcal{L}\left(\mathbf{T}^{2}\right) \backslash \mathcal{F}\left(\mathbf{T}^{2}\right)$ is $C^{2}$-proper.

2. The action of $\operatorname{Diff}\left(\mathbf{T}^{2}\right)$ on $\mathcal{F}_{1}\left(\mathbf{T}^{2}\right)$ is ergodic.

We use the word ergodic because, even if no measure is involved in this infinite dimensional context, it is nevertheless a good description of the situation.

The last part is devoted to the set $\mathcal{L}_{T}(M)$ of metrics with trivial isometry 
group. This set is dense in $\mathcal{L}(M)$ and we want to know whether it is an open set. Indeed, this is the case when $\operatorname{Diff}(M)$ acts properly. Typical examples are the Klein bottle (cf. corollary 3.5) or a 3-manifold not covered by $\mathbb{R}^{3}$ (cf. corollary 2.10). Although $\operatorname{Diff}\left(\mathbf{T}^{2}\right)$ does not act properly, $\mathcal{L}_{T}\left(\mathbf{T}^{2}\right)$ is also open in $\mathcal{L}\left(\mathbf{T}^{2}\right)$. However, in dimension greater than two, $\mathcal{L}_{T}(M)$ may not be open in $\mathcal{L}(M)$. A typical example is given by compact quotients of $P S L(2, \mathbb{R})$.

This article is a part of my doctoral dissertation ([Mo]) written under the supervision of Jacques Lafontaine. I wish to thank him for his attention throughout this work. I also wish to thank Abdelghani Zeghib, for the discussions we had and his suggestions, and Yves Carrière who kindly fixed the proof of lemma 2.2. I thank also the referees for their constructive remarks.

\section{The Riemannian situation}

For any (pseudo) metric $g$, the isometry group $\operatorname{Is}(M, g)$ is a Lie group (MyersSteenrood). It is compact when $M$ is compact and $g$ Riemannian: indeed the set of isometries is equicontinuous. With the same kind of arguments, it can be proved that the natural action of $\operatorname{Diff}(M)$ on the space of Riemannian metrics is proper. In particular, the orbits are closed.

A deeper result of D. Ebin is the slice theorem. It roughly says that any orbit of this action has a $\operatorname{Diff}(M)$-invariant tubular neighborhood. An important consequence is the (lower) semi-continuity of the cardinal and the dimension of Is $(M, g)$ with respect to $g$ (cf. theorem 8.1 of [Eb]). In particular the set of metrics without isometries is open.

We shall study all these questions: properness of the action of $\operatorname{Diff}(M)$, behavior of the orbits, properties of the set of isometry-free metrics, in the Lorentzian case, and obtain properties very different to those in the Riemannian case.

\section{Approximately stable foliations}

The action of $\operatorname{Diff}(M)$ on $\mathcal{L}(M)$ is proper (for a given topology) if for any compact subsets $K$ and $L$ of $\mathcal{L}(M)$ the set $\left\{\varphi \in \operatorname{Diff}(M) \mid \varphi^{*} K \cap L \neq \emptyset\right\}$ is compact. In fact, as it is more convenient to work with sequences rather than subsets, we will characterize the non-properness, for the $C^{k}$-topology, by the existence of two $C^{k}$ convergent sequences of metrics, $\left(h_{n}\right)_{n \in \mathbb{N}}$ and $\left(g_{n}\right)_{n \in \mathbb{N}}$, and of a non-equicontinuous (i.e. not lying in a compact subset) sequence of diffeomorphisms $\left(\varphi_{n}\right)_{n \in \mathbb{N}}$ such that $\varphi_{n}^{*} h_{n}=g_{n}$. We will call such a triple of sequences a $C^{k}$ approximately isometric system. We can view the approximately isometric systems as generalizations of the non-equicontinuous sequences of isometries. To understand the non-properness of the action we study the dynamic of those systems. Let us start with the definition (see $[\mathrm{Ze} 4]$ ) of an approximately stable vector (relative to a sequence of diffeomor- 
phisms), which will be the key notion of this section.

Definition 2.1. Let $\left(\varphi_{n}\right)_{n \in \mathbb{N}}$ be a sequence of diffeomorphisms.

- A vector $v \in T_{x} M$ is said to be approximately stable if there exists a convergent sequence $\left(v_{n}\right)_{n \in \mathbb{N}}$ with limit $v$, such that the sequence $\left(D \varphi_{n}\left(v_{n}\right)\right)_{n \in \mathbb{N}}$ is bounded. We will denote by $A S\left(\varphi_{n}\right)$ the set of approximately stable vectors of $\left(\varphi_{n}\right)_{n \in \mathbb{N}}$ and by $A S\left(x,\left(\varphi_{n}\right)\right)$ its intersection with $T_{x} M$.

We say that $v$ is strongly approximately stable if in addition $D \varphi_{n}\left(v_{n}\right) \rightarrow 0$.

- A vector $v \in T_{x} M$ is called punctually approximately stable if in addition the sequence $\left(v_{n}\right)_{n \in \mathbb{N}}$ stays in the same tangent vector space $T_{x} M$. We will denote by $P A S\left(\varphi_{n}\right)$ the set of punctually approximately stable vectors of $\left(\varphi_{n}\right)_{n \in \mathbb{N}}$ and by $P A S\left(x,\left(\varphi_{n}\right)\right)$ its intersection with $T_{x} M$.

The goal of this section is to prove the following theorem. It is a generalization of the main theorem of the article [Ze4] of A. Zeghib, which describes the dynamical properties of the non-equicontinuous sequences of Lorentzian isometries. If we compare the statements we see that the dynamical properties are mainly the same. It gives us a "localization" of the non-properness.

Theorem 2.2. Let $\left(h_{n}, g_{n}, \varphi_{n}\right)$ be a $C^{k}$ approximately isometric system (with $k \geq$ 2) on a compact manifold $M$ and let $g_{\infty}$ be the limit of $\left(g_{n}\right)_{n \in \mathbb{N}}$. Then there exists a subsequence $\left(\phi_{n}\right)_{n \in \mathbb{N}}$ of $\left(\varphi_{n}\right)_{n \in \mathbb{N}}$ such that the set of approximately stable vectors $A S\left(\phi_{n}\right)$ is the tangent bundle of a codimension 1 Lipschitz foliation, called the approximative stable foliation of $\left(\phi_{n}\right)_{n \in \mathbb{N}}$. The leaves of the foliation are geodesic and lightlike relatively to $g_{\infty}$.

Replacing $\left(\phi_{n}\right)_{n \in \mathbb{N}}$ by a subsequence if necessary, we can assume that the same is true for $\left(\phi_{n}^{-1}\right)_{n \in \mathbb{N}}$ (the foliation $A S\left(\phi_{n}^{-1}\right)$ will be geodesic and lightlike relatively to $\left.h_{\infty}\right)$. In this case, if $v \in T M \backslash A S\left(\phi_{n}\right)$, then $D \phi_{n} v$ tends to $\infty$, and converges projectively to $A S^{\perp}\left(\phi_{n}^{-1}\right)$. The convergence is uniform on compact subsets of $T M \backslash$ $A S\left(\phi_{n}\right)$.

Moreover, if $\left(\sigma_{n}\right)_{n \in \mathbb{N}}$ and $\left(\sigma_{n}^{\prime}\right)_{n \in \mathbb{N}}$ are convergent sequences of functions on $M$ whose limits are respectively $\sigma$ and $\sigma^{\prime}$ and such that $\sigma_{n}^{\prime}=\sigma_{n} \circ \varphi_{n}$, then $\sigma^{\prime}$ is constant along $A S^{\perp}\left(\phi_{n}\right)$ (and $\sigma$ along $A S^{\perp}\left(\phi_{n}^{-1}\right)$ ).

Demonstration. We start with the following essential proposition. It has been inspired by proposition 6.13 in [Eb] which shows that the orbits of the action of $\operatorname{Diff}(M)$ on the manifold of Riemannian metrics are closed.

Proposition 2.3. Let $M$ be a connected manifold (not necessarily compact), let $\left(h_{n}\right)_{n \in \mathbb{N}}$ be a $C^{k}$-convergent sequence of pseudo-Riemannian metrics on $M$ whose limit is $h_{\infty}(k \geq 2)$, and let $\left(\varphi_{n}\right)_{n \in \mathbb{N}}$ be a sequence of diffeomorphisms of $M$ such that $\left(\varphi_{n}^{*} h_{n}\right)_{n \in \mathbb{N}}$ is $C^{k}$-convergent and tends to $g_{\infty}$. Let us suppose there exist a convergent sequence $\left(x_{n}\right)_{n \in \mathbb{N}}$ of points of $M$ such that the sequence $\left(\varphi_{n}\left(x_{n}\right)\right)_{n \in \mathbb{N}}$ 
is convergent, and a real number $C>0$ such that $\forall n \in \mathbb{N},\left|D \varphi_{n}\left(x_{n}\right)\right|<C$. Then the sequence $\left(\varphi_{n}\right)_{n \in \mathbb{N}}$ admits a $C^{k}$-convergent subsequence.

The notation |.| refers to any fixed Riemannian metric. We need to suppose that the sequence $\left(\varphi_{n}\left(x_{n}\right)\right)_{n \in \mathbb{N}}$ is convergent to avoid situations like translations in vector spaces where every point is sent to infinity with bounded derivative. This is a typically non compact phenomenon. Hence, if we suppose $M$ compact we can simplify the statement.

Proof. Denote by $x$ the limit of $\left(x_{n}\right)_{n \in \mathbb{N}}$ and by $y$ the limit of $\left(\varphi_{n}\left(x_{n}\right)\right)_{n \in \mathbb{N}}$. Then the hypothesis $\left|D \varphi_{n}\left(x_{n}\right)\right|<C$ induces the existence of a convergent sequence of frames $\left(r_{n}\right)_{n \in \mathbb{N}}$ above $\left(x_{n}\right)_{n \in \mathbb{N}}$, of limit $r_{x}$, such that, restricting to a subsequence if necessary,

$$
r_{n}^{\prime}=D \varphi_{n} \cdot r_{n} \underset{n \rightarrow \infty}{\longrightarrow} r_{y}^{\prime} .
$$

The frames $r_{x}$ and $r_{y}^{\prime}$ define a linear map $L$ from $T_{x} M$ to $T_{y} M$ such that $D \varphi_{n}\left(x_{n}\right)$ tends to $L$.

Next we want to define a map $\psi: M \rightarrow M$ such that a sub-sequence of $\left(\varphi_{n}\right)_{n \in \mathbb{N}}$ converges to $\psi$. Of course, we put $\psi(x)=y$. Thanks to the following lemma, we are going to extend $\psi$ to a neighborhood of $x$. We denote by $\mathrm{e}_{n}$ (resp. $\mathrm{e}_{n}^{\prime}$ ) the exponential map of $h_{n}\left(\right.$ resp. of $\left.\varphi_{n}^{*} h_{n}\right)$ and by $\mathrm{e}\left(\right.$ resp. $\left.\mathrm{e}^{\prime}\right)$ that of $h_{\infty}$ (resp. $\left.g_{\infty}\right)$.

Lemma 2.4. Let $\left(h_{n}\right)_{n \in \mathbb{N}}$ be a $C^{k}$-convergent sequence of pseudo-Riemannian metrics whose limit is $h_{\infty}$. Then there exists an open neighborhood $U$ of the zero section of TM on which the exponential maps of the metrics $h_{n}$ are all defined. Furthermore the sequence $\left(\mathrm{e}_{n}\right)_{n \in \mathbb{N}}$ converges $C^{k-1}$ to $\mathrm{e}$ on every compact subset of $U$.

Proof. The $C^{k-1}$ convergence comes from the fact that the exponential maps are solutions of differential equations whose coefficients, which are the Christoffel symbols, converge $C^{k-1}$. We still have to check that the exponential maps are all defined at each point on non trivial subsets of the tangent spaces. To prove this, we adapt proposition 2.1 of A. Romero and M. Sanchez in [R-S]. Let $v$ be a tangent vector to $M,\left(a_{n}\right)_{n \in \mathbb{N}}$ and $\left(b_{n}\right)_{n \in \mathbb{N}}$ be two sequences of real numbers such that $\left[a_{n}, b_{n}\right]$ is the domain of $\gamma_{n}$ the $h_{n}$-geodesic stemming from $v$, and $a$ and $b$ such that $[a, b]$ is that of $\gamma$ the $h_{\infty}$-geodesic. It is sufficient to show that

$$
\lim \sup a_{n} \leq a<0<b \leq \liminf b_{n} .
$$

Put $A=\left\{t \in\left[0, b\left[\mid t<b_{n}\right.\right.\right.$ for all $n$ but a finite number and $\gamma_{n}^{\prime}(t)$ converge to $\left.\gamma^{\prime}(t)\right\}$. Using that solutions of differential equations depend continuously on the initial conditions and on the coefficients of the equations, we see that $A$ is open and closed in $[0, b[$ and therefore $A=[0, b[$.

Let us continue the proof of proposition 2.3. The following relation holds: 
Relation 2.5. $\varphi_{n} \circ \mathrm{e}_{n}^{\prime}=\mathrm{e}_{n} \circ D \varphi_{n}$.

According to lemma 2.4, there is an open subset $U$ of $T M$ on which $\mathrm{e}_{n}\left(\varphi_{n}\left(x_{n}\right)\right)$ tends to $\mathrm{e}(y)$ and $\mathrm{e}_{n}^{\prime}\left(x_{n}\right)$ tends to $\mathrm{e}^{\prime}(x)$. Restricting to an open subset if necessary, we can assert that $\left(\mathrm{e}_{n}^{\prime}\left(x_{n}\right)\right)^{-1}$ tends to $\left(\mathrm{e}^{\prime}(x)\right)^{-1}$. As $x_{n}$ tends to $x$, there exists a neighborhood $V$ of $x$ such that, for $n$ sufficiently large, $\left(\mathrm{e}_{n}^{\prime}\left(x_{n}\right)\right)^{-1}$ is well defined on it.

Taking $x^{\prime} \in V$, one finds

$$
\begin{aligned}
\varphi_{n}\left(x^{\prime}\right) & =\varphi_{n}\left(\mathrm{e}_{n}^{\prime}\left(x_{n}\right) \circ\left(\mathrm{e}_{n}^{\prime}\left(x_{n}\right)\right)^{-1}\left(x^{\prime}\right)\right) \\
& =\mathrm{e}_{n}\left(D \varphi_{n}\left(x_{n}\right) \circ\left(\mathrm{e}_{n}^{\prime}\left(x_{n}\right)\right)^{-1}\left(x^{\prime}\right)\right),
\end{aligned}
$$

using relation 2.5. However, the sequence of linear maps $\left(D \varphi_{n}\left(x_{n}\right)\right)_{n \in \mathbb{N}}$ converges to $L$ and therefore

$$
\lim _{n \rightarrow \infty} \varphi_{n}\left(x^{\prime}\right)=\mathrm{e}(y) \circ L \circ\left(\mathrm{e}^{\prime}(x)\right)^{-1}\left(x^{\prime}\right),
$$

which we set to be $\psi\left(x^{\prime}\right)$. By construction, we see that the sequence $\left(\varphi_{n}\right)_{n \in \mathbb{N}}$ converges, $C^{k-1}$, to $\psi$ and that $\psi$ is a local $C^{\infty}$ diffeomorphism. Using the fact that $D \varphi_{n}\left(x^{\prime}\right)$ tends to $D \psi\left(x^{\prime}\right)$, we repeat the same construction to extend along the geodesics to the whole manifold. We have constructed a local $C^{\infty}$ diffeomorphism $\psi$ on $M$. The sequence $\left(\varphi_{n}\right)_{n \in \mathbb{N}}$ converges, a priori, only $C^{k-1}$ to $\psi$.

Next we look at the sequence of the connections, which is $C^{k-1}$ convergent. We reproduce the computations done by D. Ebin ([Eb], proposition 6.13). In local coordinates we represent $\varphi_{n}$ by $\varphi_{n}^{i}\left(x^{1}, \ldots, x^{m}\right)$. Let ${ }_{n} \Gamma_{i j}^{l}\left(\right.$ resp. $\left.{ }_{n} \Gamma^{\prime l}{ }_{i j}\right)$ denote the Christoffel symbols of the metric $h_{n}$ (resp. of $\left.\varphi_{n}^{*} h_{n}\right)$. We have

$$
{ }_{n} \Gamma_{i j}^{\prime l}=\left(\frac{\partial \varphi_{n}^{i}}{\partial x^{r}}\right)^{-1}\left(\frac{\partial \varphi_{n}^{j}}{\partial x^{s}}\right)^{-1}{ }_{n} \Gamma_{r s}^{t}\left(\frac{\partial \varphi_{n}^{l}}{\partial x^{t}}\right)-\left(\frac{\partial^{2} \varphi_{n}^{l}}{\partial x^{s} \partial x^{r}}\right)\left(\frac{\partial \varphi_{n}^{i}}{\partial x^{s}}\right)^{-1}\left(\frac{\partial \varphi_{n}^{j}}{\partial x^{r}}\right)^{-1}
$$

where "-1" indicates matrix inverse. The convergence $C^{k-1}$ of the sequences of Christoffel symbols and the convergence $C^{k-2}$ of the first order derivatives of $\varphi_{n}$ imply the convergence $C^{k-2}$ of the second order derivatives, hence the convergence $C^{k}$ of the sequence of diffeomorphisms $\left(\varphi_{n}\right)_{n \in \mathbb{N}}$.

We still have to show that $\psi$ is a diffeomorphism, for this we use the symmetry of the problem. Using that, asymptotically, the $\varphi_{n}$ permute the induced volume forms of the metrics $h_{\infty}$ and $g_{\infty}$, we see that the sequence $\left(\varphi_{n}^{-1}\right)_{n \in \mathbb{N}}$ satisfies also the hypothesis of the proposition and so we obtain the existence of $\psi^{-1}$. This conclude the proof of proposition 2.3

\section{Remarks.}

1. Proposition 2.3 can be used to prove that on the space of Riemannian metrics, the action is $C^{2}$-proper: indeed when $M$ is compact and if the metrics are Riemannian, the hypotheses of the proposition are always satisfied. 
2. This proposition has an interesting interpretation: it asserts that the sequences of diffeomorphisms we are interested in are nowhere or everywhere divergent.

As in the proof of A. Zeghib, we first consider the problem in the linear case. Let us start the proof of theorem 2.2 with the following evident lemma whose proof is left to the reader.

Lemma 2.6. If $\left(H_{n}\right)_{n \in \mathbb{N}}$ is a convergent sequence of quadratic forms of signature $(m-1,1)$ on $\mathbb{R}^{m}$ whose limit is $H$, then there exists a sequence of linear maps $\left(M_{n}\right)_{n \in \mathbb{N}}$ of limit Id such that $H_{n}={ }^{t} M_{n} H M_{n}$.

This lemma shows that the linear approximately isometric systems will have the same behavior as the non-equicontinuous (non bounded) sequences of linear isometries. As a consequence, corollary 4.3 from [Ze4] is immediately transposable, and thus we have the following.

Proposition 2.7. Let $\left(H_{n}, G_{n}, M_{n}\right)$ be a linear approximately isometric system on $\mathbb{R}^{m}$. Then there exists a subsequence $\left(N_{n}\right)_{n \in \mathbb{N}}$ such that $A S\left(0, N_{n}\right)$ is a lightlike hyperplane and moreover $A S^{\perp}\left(0,\left(N_{n}\right)\right)$ is the set of strongly approximately stable vectors. Moreover, there exist $C>0$ and a sequence of hyperplanes $\left(P_{n}\right)_{n \in \mathbb{N}}$ such that we have the following uniformity condition: $\forall n \in \mathbb{N},\left|N_{\left.n\right|_{P_{n}}}\right|<C$.

Thanks to a trivialization of the tangent (measurable but continuous at the neighborhood of the point considered) we associate to each diffeomorphism a matrix field, $C_{n}(x)$. We have

$$
P A S\left(x,\left(\varphi_{n}\right)\right)=P A S\left(0,\left(C_{n}(x)\right)\right)=A S\left(0,\left(C_{n}(x)\right)\right) .
$$

Proposition 2.3 tells us that if the sequence $\left(\varphi_{n}\right)_{n \in \mathbb{N}}$ is non-equicontinuous then, for all $x$, the sequence $\left(C_{n}(x)\right)_{n \in \mathbb{N}}$ is not bounded. By a diagonal process, we prove that there exists a subsequence $\left(\phi_{n}\right)_{n \in \mathbb{N}}$ of $\left(\varphi_{n}\right)_{n \in \mathbb{N}}$ and a dense subset $M^{\prime}$ of $M$ such that, for all $x \in M^{\prime}, P A S\left(x, \phi_{n}\right)$ is a hyperplane of $T_{x} M$ that we will denote by $P_{x}$. Keeping the same notations for the exponential maps as before, we can prove the following.

Proposition 2.8. For all $x \in M^{\prime}$, there exists a neighborhood $V_{x}$ of 0 in $T_{x} M$ such that the hypersurface $\mathcal{I}_{x}=\mathrm{e}^{\prime}\left(P_{x} \cap V_{x}\right)$ is geodesic and such that for all $y \in \mathcal{I}_{x}$, $T_{y} \mathcal{I}_{x}=A S\left(y, \phi_{n}\right)$.

Proof. Let $\left(P_{n}\right)_{n \in \mathbb{N}}$ be the sequence of hyperplanes in $T_{x} M$ given by proposition 2.7 applied to the $C_{n}(x)$. We are going to use the fact (cf. lemma 2.4) that the sequences of exponential maps converge. Let $y$ be a point of $\mathcal{I}_{x}$, we first write the differential of the $\phi_{n}$ at this point using the exponential maps of the metrics $g_{n}$ 
that we denote, as before, by $\mathrm{e}_{n}^{\prime}$.

$$
\begin{aligned}
D_{y} \phi_{n} & =D_{\left(\mathrm{e}_{n}^{\prime}(x)\right)^{-1}(y)}\left(\phi_{n} \circ \mathrm{e}_{n}^{\prime}(x)\right) \circ D_{y}\left(\mathrm{e}_{n}^{\prime}(x)\right)^{-1} \\
& =D_{x}\left(\mathrm{e}_{n}\left(\phi_{n}(x)\right) \circ \phi_{n}\right) \circ D_{y}\left(\mathrm{e}_{n}^{\prime}(x)\right)^{-1}
\end{aligned}
$$

according to relation 2.5. We set $P_{n}^{\prime}=T_{y}\left(\mathrm{e}_{n}^{\prime}\left(P_{n} \cap V_{n}\right)\right)$, with $V_{n}$ a bounded neighborhood of 0 in $T_{x} M$ inside the domain in which $\mathrm{e}_{n}^{\prime}$ is defined. It is clear that $P_{n}^{\prime} \rightarrow T_{y} \mathcal{I}_{x}$, but also that $D_{y}\left(\mathrm{e}_{n}^{\prime}(x)\right)^{-1} \cdot P_{n}^{\prime}=P_{n}$. Consequently, as the sequence $\left(\mathrm{e}_{n}^{\prime}\right)_{n \in \mathbb{N}}$ is convergent, $\left|D \phi_{n \mid P_{n}^{\prime}}\right|$ is bounded. Hence, $T_{y} \mathcal{I}_{x} \subset A S\left(y, \phi_{n}\right)$. However, thanks to proposition 2.3 (following the proof of the fact 6.4 of [Ze4]), we can prove that $A S\left(y, \phi_{n}\right)$ is at most a hyperplane therefore we have equality.

Now, we have to prove that this surface is geodesic. Let $c$ be a path on $\mathcal{I}_{x}$ between $x$ and $y$. The neighborhood $V_{n}$ being bounded, we see that the sequence $\left(d_{n}\right)_{n \in \mathbb{N}}$, defined by $d_{n}=\phi_{n} \circ c$, is with $C^{1}$ bounded variation. We denote by $\tau_{n}$ the parallel transport along $c$ for the metric $h_{n}$ and by $\tau_{n}^{\prime}$ the parallel transport along $d_{n}$ for the metric $g_{n}$. Finally, we denote by $P_{n}^{\prime \prime}$ the image of $P_{n}$ by $\tau_{n}$ and by $P^{\prime \prime}$ the limit of this sequence. We have:

$$
D_{y} \phi_{n}=\tau_{n}^{\prime} \circ D_{x} \phi_{n} \circ \tau_{n}^{-1} .
$$

Knowing that the sequence of paths has bounded variation and the sequences of metrics are $C^{1}$ convergent, it becomes clear that we have a new sequence of stable hyperplanes that is $P^{\prime \prime}$ is approximately stable. Therefore $P^{\prime \prime}=A S\left(y, \phi_{n}\right)$ and so $P^{\prime \prime}=T_{y} \mathcal{I}_{x}$. The surface is therefore geodesic.

The size of the neighborhood $V_{x}$ depends essentially on the domains on which the exponential maps are defined. Moreover, two plaques $\mathcal{I}_{x}$ and $\mathcal{I}_{y}$ are either disjoint or tangent. Thus, the hypotheses of the following lemma (cf. [Ze4] and [Ze3]) are fulfilled.

Lemma 2.9. Let $M$ be a compact manifold endowed with a torsion free connection and an auxiliary norm |.| on TM. Let $M^{\prime}$ be a dense subset of $M$ and suppose given a real number $r$ and for $x \in M^{\prime}$ a hyperplane $P_{x} \subset T_{x} M$ and let $\mathcal{I}_{x, r}=$ $\exp \left(P_{x} \cap B_{x}(r)\right)$, where $B_{x}(r)$ is the ball of $T_{x} M$ centered at 0 and with radius $r$. Also, suppose that $\mathcal{I}_{x, r}$ is geodesic and that if two plaques $\mathcal{I}_{x, r}$ and $\mathcal{I}_{y, r}$ intersect at some point, then they are tangent at that point (and hence by geodesibility, the intersection $\mathcal{I}_{x, r} \cap \mathcal{I}_{y, r}$ is open in both $\mathcal{I}_{x, r}$ and $\left.\mathcal{I}_{y, r}\right)$. Then the geodesic plaques $\mathcal{I}_{x, r}$ extend to a Lipschitz geodesic foliation of $M$.

Now, to finish the proof of the first part, we have to show that the tangent to this foliation is still approximately stable. Moreover, we can prove that $A S\left(\phi_{n}\right)=$ $P A S\left(\phi_{n}\right)$, and also that the set of strongly approximately stable vectors is the tangent bundle to the 1 dimensional geodesic lightlike foliation $A S^{\perp}\left(\phi_{n}\right)$. The proof of those three points following exactly Zeghib's proof, we refer to [Ze4]. 
We only give the main idea of the proof of the second part of the theorem. It is exactly the same as the original case (proposition 9.5 of [Ze4]). It uses the fact that if $v \notin A S\left(\phi_{n}\right)$ then there exists a sequence of real numbers $\left(\alpha_{n}\right)_{n \in \mathbb{N}}$ and a sequence of vectors $\left(u_{n}\right)_{n \in \mathbb{N}}$ such that $D \phi_{n} v=\alpha_{n} u_{n}$. Of course, $\alpha_{n} \rightarrow \infty$ and therefore $\left|D \phi_{n}^{-1}\left(u_{n}\right)\right| \rightarrow 0$. The limit of the sequence of vectors is consequently in $A S^{\perp}\left(\phi_{n}^{-1}\right)$.

Let us show the last part of the theorem. Let $x$ be a point of $M$, and let $\left(v_{n}\right)_{n \in \mathbb{N}}$ be a convergent sequence of vectors which tends to $v$ and such that $D \phi_{n}\left(v_{n}\right) \rightarrow 0$, i.e. $v \in A S^{\perp}\left(\phi_{n}\right)$. Let us consider $\Delta_{n}=\sigma_{n}^{\prime}\left(\mathrm{e}_{n}(x) \cdot v_{n}\right)-\sigma_{n}^{\prime}(x)$, we have

$$
\begin{aligned}
\Delta_{n} & =\sigma_{n}\left(\phi_{n}\left(\mathrm{e}_{n}(x) \cdot v_{n}\right)\right)-\sigma_{n}\left(\phi_{n}(x)\right) \\
& =\sigma_{n}\left(\mathrm{e}_{n}^{\prime}\left(D \phi_{n} v_{n}\right)\right)-\sigma_{n}\left(\phi_{n}(x)\right),
\end{aligned}
$$

using relation 2.5. If we take $\left(v_{n}\right)_{n \in \mathbb{N}}$ such that $v_{n} \rightarrow v$ and $D \phi_{n}\left(v_{n}\right) \rightarrow 0$, we have

$$
\lim _{n \rightarrow \infty} \mathrm{e}_{n}^{\prime}\left(D \phi_{n}\left(v_{n}\right)\right)=\lim _{n \rightarrow \infty} \phi_{n}(x)
$$

therefore

$$
\sigma_{n}\left(\mathrm{e}_{n}^{\prime}\left(D \phi_{n} v_{n}\right)\right)-\sigma_{n}\left(\phi_{n}(x)\right) \underset{n \rightarrow \infty}{\longrightarrow} 0
$$

and therefore $\Delta_{n}$ also converges to 0 . However, $\sigma_{n}^{\prime}\left(\mathrm{e}_{n}(x) \cdot v_{n}\right)$ converges to $\sigma^{\prime}(\mathrm{e}(x) . v)$ and $\sigma_{n}^{\prime}(x)$ converges to $\sigma^{\prime}(x)$. Therefore, we can assert that $\sigma^{\prime}$ is constant along the $g_{\infty}$-geodesic stemming from $v$, i.e. $A S^{\perp}\left(\phi_{n}\right)$ according to what preceded.

In [D-Gr], G. D'Ambra and M. Gromov conjectured that if $M$ is compact and simply connected then the action is $C^{2}$-proper. Theorem 2.2 solves the 3 dimensional case.

Corollary 2.10. Let $M$ be a 3 dimensional compact manifold not covered by $\mathbb{R}^{3}$. Then the action of the group of diffeomorphisms on $\mathcal{L}(M)$ is proper for the $C^{2}$ topology.

Proof. According to [Ze3], these manifolds do not possess lightlike geodesic foliations of codimension 1 therefore theorem 2.2 finishes the proof.

This statement can be found without proof in [Ze4].

Since any Lorentzian manifold possess a negative line-field, we see that the metrics $h_{\infty}$ and $g_{\infty}$ of theorem 2.2 have two nowhere collinear line-fields. It trivially gives a topological obstruction. For example, thanks to the famous theorem of J. F. Adams (see $[\mathrm{Ad}]$ ) about vector fields on spheres, we have:

Corollary 2.11. If $n \equiv 2 \bmod 4$, then the action of $\operatorname{Diff}\left(S^{n-1}\right)$ on $\mathcal{L}\left(S^{n-1}\right)$ is proper. 


\section{On the torus}

\subsection{Localization of the non properness}

There exist (flat) metrics on the torus with non compact isometry group, consequently the action of the group of diffeomorphisms of the torus on $\mathcal{L}\left(\mathbf{T}^{2}\right)$ is not proper. A priori, the approximately isometric systems $\left(h_{n}, g_{n}, \varphi_{n}\right)$ can have a very different shape. The point is to understand it.

Even if every Lorentzian surface admits two geodesic lightlike foliations of (co)dimension 1 , theorem 2.2 will enable us to localize precisely the non properness of the action by showing that the limit metrics $h_{\infty}$ and $g_{\infty}$ are flat. We first show the following result.

Theorem 3.1. A Lorentzian metric on the torus $\mathbf{T}^{2}$ whose curvature is constant along one of its lightlike foliations is flat.

Let us remark that, of course, the lightlike condition is necessary as the case of the Clifton-Pohl torus shows (cf. [C-R]). There also exist local counterexamples: if we take the metric $h(x, y)=d x d y+2 x y^{2} d x^{2}$, its curvature is $K(x, y)=-x$ which is constant along the lightlike direction $\partial_{y}$.

Proof. We call $\mathcal{F}$ the foliation along which the curvature is constant. According to the Gauss-Bonnet theorem [B-N], the curvature has to vanish somewhere. Therefore there exists a leaf $F$ of $\mathcal{F}$ such that the curvature, $K$, vanishes along $F$. Two cases have to be considered: the leaf can be compact or not. Let us suppose the leaf is not compact. If the foliation is conjugated to a linear foliation then $F$ is everywhere dense and $h_{\infty}$ is clearly flat. If this is not the case, $\mathcal{F}$ has some compact leaves $(\mathrm{cf}$. $[\mathrm{H}-\mathrm{H}])$. We can thus reduce the problem to a foliation on an annulus. Therefore we can state (cf. theorem 4.2.15 [H-H]) that the leaf $F$ is going to accumulate on two compact leaves. We denote by $A$ the Lorentzian annulus delimited by those leaves. All the leaves of $\mathcal{F}$ contained in $A$ are going to accumulate on the boundary of $A$. Therefore this annulus is flat.

Lemma 3.2. If $A$ is a flat Lorentzian annulus with lightlike boundary and $\mathcal{F}$ is the lightlike foliation tangent to the boundary, then all the leaves of $\mathcal{F}$ are compact.

Proof. Let $\widetilde{A}$ be the universal cover of $A$ (topologically $\widetilde{A}$ is a stripe) and $\gamma$ be a generator of $\pi_{1}(A)$. The flat Lorentzian manifold $\widetilde{A}$ is developed by a local diffeomorphism $D: \widetilde{A} \longrightarrow \mathbb{R}^{2}$ (where $\mathbb{R}^{2}$ is endowed with the Lorentzian metric $x y)$ which satisfies the following equivariant condition :

$$
D \circ \gamma=\gamma^{\prime} \circ D
$$

where $\gamma^{\prime}$ is a Lorentzian isometry (the holonomy of $\gamma$ ). The foliation $\mathcal{F}$ is lifted to a foliation $\widetilde{\mathcal{F}}$ of $\widetilde{A}$. We can suppose that $\widetilde{\mathcal{F}}$ is the pull-back by $D$ of the foliation 
of $\mathbb{R}^{2}$ by horizontal lines.

Translating the situation if necessary, we have two cases:

a) $\gamma^{\prime}=\left(\begin{array}{cc}\lambda & 0 \\ 0 & 1 / \lambda\end{array}\right)$, with $\lambda \in \mathbb{R}^{*}$

b) $\gamma^{\prime}$ is an horizontal translation.

The case a) is impossible; in fact, the continuous function $f(x, y)=x y$ is $\gamma^{\prime}$ invariant and, on the closed superior and inferior half-planes, its only possible local extremum is 0 . According to the condition $(*)$ and the fact that $D$ is a local diffeomorphism, the continuous function $g=f \circ D$ is $\gamma$-invariant and its only local extremum is 0 . Therefore, it descends to $A$ as a constant function everywhere equal to 0 , which is not possible.

Therefore the only remaining case is $\mathrm{b}$ ). In this case, the annulus $A$ (for the same affine structure) admits a flat Riemannian metric, which implies that $D$ is a diffeomorphism (the structure is complete as $A$ is compact). Consequently, $\widetilde{A}$ can be directly seen as a horizontal stripe of $\mathbb{R}^{2}$ and we obtain $A$ by quotienting by a horizontal translation. Hence, all the leaves of $\mathcal{F}$ are closed.

We deduce from this that if $\mathcal{F}$ has no dense leaf (we can assume this) then a leaf $F$, along which the curvature is zero, has to be compact. Moreover, let us show that $F$ is geodesically complete. Let $\varphi$ be a germ of diffeomorphisms at $0 \in \mathbb{R}$ which generates the holonomy of $F$. According to the article of Y. Carrière and L. Rozoy [C-R], the completeness of $F$ is characterized by the divergence of the two series $\sum_{0}^{ \pm \infty} \varphi^{\prime}(0)^{k}$ i.e. by $\left|\varphi^{\prime}(0)\right|=1$. But, if $\left|\varphi^{\prime}(0)\right| \neq 1$ then $F$ is attractive (or repulsive according to the choice of an orientation). A leaf attracted by $F^{\prime}$ would be non compact and the curvature would vanish along it. That contradicts what we just proved. Hence every leaf of $\mathcal{F}$ along which the curvature vanishes is a compact and complete geodesic.

If the curvature is not constant around $F$ we can consider, using a transverse curve, the closest leaf of $\mathcal{F}$ with zero curvature. Again we obtain a Lorentzian annulus $A^{\prime}$ (we can also take the closure of a connected component of $\mathbf{T}^{2} \backslash\left\{K^{-1}(0)\right\}$ ). The connected components of the boundary of $A^{\prime}$ are complete lightlike geodesics and the curvature does not vanish inside $A^{\prime}$. We are going to use the following version of the Gauss-Bonnet theorem.

Lemma 3.3. Let $A$ be a Lorentzian annulus with lightlike boundary $\Gamma=\gamma_{1} \cup \gamma_{2}$. Let $\gamma_{i}:[0,1] \rightarrow \Gamma, i \in\{1,2\}$ be a parametrization of the boundary. Let $Z_{i}$ be a vector field along $\gamma_{i}$, tangent to $\gamma_{i}$ and parallel. We define $\lambda_{i}$ as the proportionality coefficient between $Z_{i}(0)$ and $Z_{i}(1)$. Then we have:

$$
\int_{A} K d v_{h}=\ln \left(\lambda_{1} / \lambda_{2}\right) .
$$

If the boundary is made of complete geodesics, we have:

$$
\int_{A} K d v_{h}=0
$$


Proof. Let $X$ be a nowhere vanishing lightlike vector field tangent to the boundary, $X^{0}$ be another lightlike vector field such that $h\left(X, X^{0}\right)=1$ and $\omega$ be the 1 -form defined by $\omega(v)=h\left(\nabla_{v} X, X^{0}\right)$, where $\nabla$ is the Levi-Civita connection of the metric. Then:

$$
\begin{aligned}
d \omega(v, u)= & v \cdot \omega(u)-u \cdot \omega(v)-\omega([v, u]) \\
= & v \cdot h\left(\nabla_{u} X, X^{0}\right)-u \cdot h\left(\nabla_{v} X, X^{0}\right)-h\left(\nabla_{[v, u]} X, X^{0}\right) \\
= & h\left(\nabla_{v} \nabla_{u} X, X^{0}\right)+h\left(\nabla_{u} X, \nabla_{v} X^{0}\right)-h\left(\nabla_{u} \nabla_{v} X, X^{0}\right) \\
& -h\left(\nabla_{v} X, \nabla_{u} X^{0}\right)-h\left(\nabla_{[v, u]} X, X^{0}\right) \\
= & h\left(R(v, u) X, X^{0}\right)+h\left(\nabla_{u} X, \nabla_{v} X^{0}\right)-h\left(\nabla_{v} X, \nabla_{u} X^{0}\right) .
\end{aligned}
$$

Contrarily to the Riemannian case and to the case where $X$ is timelike (see [B-N]), we do not have automatically $h\left(\nabla_{u} X, \nabla_{v} X^{0}\right)=0$. Nevertheless, we still have $h\left(\nabla_{u} X, X^{0}\right)=0$ and so $h\left(\nabla_{u} X, \nabla_{v} X^{0}\right)=h\left(\nabla_{u} X, X^{0}\right) h\left(X, \nabla_{v} X^{0}\right)$. Derivating the equality $h\left(X, X^{0}\right)=1$, we obtain immediately $h\left(\nabla_{u} X, X^{0}\right)=-h\left(X, \nabla_{u} X^{0}\right)$. Therefore we have

$$
\begin{aligned}
h\left(\nabla_{u} X, \nabla_{v} X^{0}\right) & =h\left(\nabla_{u} X, X^{0}\right) h\left(X, \nabla_{v} X^{0}\right) \\
& =-h\left(X, \nabla_{u} X^{0}\right)\left(-h\left(\nabla_{v} X, X^{0}\right)\right) \\
& =h\left(\nabla_{v} X, \nabla_{u} X^{0}\right) .
\end{aligned}
$$

Hence $d \omega\left(X, X^{0}\right)=h\left(R\left(X, X^{0}\right) X, X^{0}\right)=K$ and so $d \omega=K d v_{h}$. According to Stokes' theorem, we have:

$$
\int_{A} K d v_{h}=\int_{\Gamma} \omega
$$

where $\Gamma=\gamma_{1} \cup \gamma_{2}$ is the boundary of $A$. Let $Z_{1}$ be a parallel vector field tangent to $\gamma_{1}$. Then $Z_{1}=\mu X$, and we obtain

$$
\nabla_{Z_{1}} Z_{1}=\left(Z_{1} \cdot \mu\right) X+\mu \omega\left(Z_{1}\right) X=0
$$

Thus we have $\omega\left(Z_{1}\right)=\left(-Z_{1} \cdot \mu\right) / \mu$, and therefore $\int_{\gamma_{1}} \omega=-\ln \mu(0)+\ln \mu(1)=$ $-\ln \lambda_{1}$. Doing the same with $\gamma_{2}$ yields

$$
\int_{\Gamma} \omega=\ln \left(\lambda_{2} / \lambda_{1}\right)
$$

We can now finish the proof of the theorem. On the annulus $A^{\prime}$ we had previously, we apply lemma 3.3 and find $\int_{A^{\prime}} K=0$. However $K$ does not vanish inside $A^{\prime}$ and thus we have a contradiction and therefore $K=0$ on all $\mathbf{T}^{2}$.

This result applies directly to our problem. If, as in the introduction, we denote by $\mathcal{F}\left(\mathbf{T}^{2}\right)$ the set of flat metrics of the torus, the following is true.

Corollary 3.4. The action of $\operatorname{Diff}\left(\mathbf{T}^{2}\right)$ on $\mathcal{L}\left(\mathbf{T}^{2}\right)-\mathcal{F}\left(\mathbf{T}^{2}\right)$ is $C^{k}$-proper, $k \geq 2$.

Proof. It is clear that the statement is a consequence of the following stronger proposition: if $\left(h_{n}, g_{n}, \varphi_{n}\right)$ is a $C^{2}$ approximately isometric system on $\mathbf{T}^{2}$, then the 
metrics $h_{\infty}$ and $g_{\infty}$ are flat. According to theorem 2.2, $A S^{\perp}\left(\varphi_{n}\right)$ and $A S^{\perp}\left(\varphi_{n}^{-1}\right)$ are lightlike Lipschitz line-fields (actually, in dimension 2 they are automatically smooth). We can apply the second part of the theorem to the curvature functions. Indeed, if we denote by $K_{n}$ the curvature of $h_{n}$ and $K_{n}^{\prime}$ the one of $g_{n}$, these functions satisfy the relation $K_{n} \circ \varphi_{n}=K_{n}^{\prime}$ and the $C^{2}$-convergence of the sequences of metrics implies the convergence of the sequences of curvature functions. Hence, the curvatures of both $h_{\infty}$ and $g_{\infty}$ are constant along a lightlike foliation. According to the previous theorem these metrics are flat.

This corollary will be of precious help to study the manifestations of the non properness of the action of $\operatorname{Diff}(M)$ on $\mathcal{L}\left(\mathbf{T}^{2}\right)$. But, on the Klein bottle, it entails the following interesting result.

Corollary 3.5. Let $K$ be the Klein bottle. The action of $\operatorname{Diff}(K)$ on $\mathcal{L}(K)$ is proper.

Proof. We are going to show that the sequences of diffeomorphisms that compose the approximately isometric systems of the torus can never descend to the Klein bottle. For this we first show the

Lemma 3.6. Let $\left(h_{n}, g_{n}, \varphi_{n}\right)$ be a $C^{2}$-approximately isometric system on $\mathbf{T}^{2}$ and let $f_{n}$ be the lift to $\mathbb{R}^{2}$ of $\varphi_{n}$. Then there exists a sequence $\left(N_{n}\right)_{n \in \mathbb{N}}$ of affine maps of $\mathbb{R}^{2}$ such that the sequence $\left(N_{n} \circ f_{n}\right)_{n \in \mathbb{N}}$ converges to the identity map.

Proof. As we permit right or left composition by diffeomorphisms, we can suppose that the lifts of $h_{\infty}$ and of $g_{\infty}$, that we denote by $H$ and $G$, are quadratic forms and even that $H=G$. We denote by $H_{n}$ (resp. $G_{n}$ ) the lift of the metric $h_{n}$ (resp. $\left.g_{n}\right)$. Of course, $f_{n}^{*} H_{n}=G_{n}$ and converges to $G$. We denote by $x_{n}$ a point of $\mathbb{R}^{2}$ that realizes the maximum of the norm of the second derivative of $H_{n}$ (seen as a map from $\mathbb{R}^{2}$ to $G L\left(2, \mathbb{R}^{2}\right)$ ). Let $M_{n}$ be the linear map $D f_{n}\left(f_{n}^{-1}\left(x_{n}\right)\right)$. We know that ${ }^{t} M_{n} H_{n}\left(x_{n}\right) M_{n}$ converges to $H$, hence from lemma 2.6, $M_{n}$ is close to an isometry of $H$. Let us choose a basis of $\mathbb{R}^{2}$ such that the metrics are given by the matrices

$$
H=\left(\begin{array}{ll}
0 & 1 \\
1 & 0
\end{array}\right) \quad \text { and } \quad H_{n}(x)=\left(\begin{array}{ll}
a_{n}(x) & b_{n}(x) \\
b_{n}(x) & c_{n}(x)
\end{array}\right)
$$

We can suppose that $M_{n}$ is close to

$$
\left(\begin{array}{cc}
1 / \lambda_{n} & 0 \\
0 & \lambda_{n}
\end{array}\right)
$$

with $\lambda_{n} \rightarrow \infty$.

As $H_{n} \rightarrow H$, for the $C^{2}$ topology, it is easy to see that $D^{2}\left({ }^{t} M_{n} H_{n}(x) M_{n}\right) \rightarrow 0$ uniformly if and only if $D^{2}\left(\lambda_{n}^{2} c_{n}\right) \rightarrow 0$ uniformly. As $f_{n}^{*} H_{n} \rightarrow H$, we have $D^{2}\left(\lambda_{n}^{2} c_{n}\left(x_{n}\right)\right) \rightarrow 0$ and as $x_{n}$ realizes the maximum of the second derivative we 
have the uniform convergence to 0 . Now we use again that $f_{n}^{*} H_{n} \rightarrow H$ to show that $D\left({ }^{t} M_{n} H_{n}\left(x_{n}\right) M_{n}\right) \rightarrow 0$ and that ${ }^{t} M_{n} H_{n}\left(x_{n}\right) M_{n} \rightarrow H$. We deduce from this that $G_{n}^{\prime}=M_{n}^{*} H_{n} \rightarrow H$ for the $C^{2}$ topology. We can define a sequence of translations $\left(\tau_{n}\right)_{n \in \mathbb{N}}$, such that $\tau_{n}\left(M_{n}^{-1}\left(x_{n}\right)\right)=0$. The sequence $G_{n}^{\prime \prime}=\tau_{n}^{*} G_{n}^{\prime}$ still converges. We denote by $N_{n}$ the map $\left(M_{n} \circ \tau_{n}\right)^{-1}$. Therefore $\left(N_{n} \circ f_{n}\right)^{*} G_{n}^{\prime \prime} \rightarrow G$. However, the sequence of the derivatives of $\left(N_{n} \circ f_{n}\right)$ at $f_{n}^{-1}\left(x_{n}\right)$ is bounded and $\left(N_{n} \circ f_{n}\right)\left(f_{n}^{-1}\left(x_{n}\right)\right)=0$. We recognize the hypothesis of proposition 2.3. Extracting a subsequence if necessary, we can say that the sequence $\left(N_{n} \circ f_{n}\right)_{n \in \mathbb{N}}$ is $C^{2}$-convergent. Its limit is an isometry of $H$ tangent to a translation and therefore it is a translation. It is not hard, now, to obtain the desired sequence.

We continue the proof of the corollary. Taking $n$ sufficiently big, we write $f_{n}=M_{n} \circ \varepsilon_{n}$ with $M_{n}$ linear and $\varepsilon_{n}$ a diffeomorphism close to the identity. Let $\sigma$ be the "antipodal" map defined by $\sigma(x, y)=(x+1 / 2,-y)$. The fact that $f_{n}$ actually descends to the Klein bottle can be written $f_{n} \circ \sigma=\sigma \circ f_{n}$. We have

$$
\begin{aligned}
M_{n} \circ \varepsilon_{n} \circ \sigma & =\sigma \circ M_{n} \circ \varepsilon_{n} \\
M_{n} \circ \varepsilon_{n} \circ \sigma \circ \varepsilon_{n}^{-1} & =\sigma \circ M_{n},
\end{aligned}
$$

these equalities being on the torus. We set $\sigma_{n}^{\prime}=\varepsilon_{n} \circ \sigma \circ \varepsilon_{n}^{-1}$. Of course, $\sigma$ and $\sigma_{n}^{\prime}$ are close. Then we compute $\left(M_{n} \circ \sigma_{n}^{\prime}-\sigma \circ M_{n}\right)(x, 0)$ and $\left(M_{n} \circ \sigma_{n}^{\prime}-\sigma \circ M_{n}\right)(0, y)$ which have to be in $\mathbb{Z}^{2}$. It gives immediately that $M_{n}$ has to be diagonal. Using again the fact that $\left(f_{n}\right)_{n \in \mathbb{N}}$ descends to the quotient and is not equicontinuous, we see that it is impossible. The sequence cannot descend to the Klein bottle.

\subsection{Action of $\operatorname{Diff}\left(\mathbf{T}^{2}\right)$ on $\mathcal{F}\left(\mathbf{T}^{2}\right)$}

As we announced in the introduction, we are going to prove that the action of the group of diffeomorphisms on the set of flat metrics of volume 1, denoted by $\mathcal{F}_{1}\left(\mathbf{T}^{2}\right)$, is ergodic (or more correctly that it has the same dynamical properties as a given ergodic action). If we want to talk about ergodicity, we first have to reduce the problem to a finite dimensional one. This reduction is done by the following proposition.

Proposition 3.7. The action of $\operatorname{Diff}_{0}\left(\mathbf{T}^{2}\right)$ on $\mathcal{F}_{1}\left(\mathbf{T}^{2}\right)$ is proper and $\mathcal{F}_{1}\left(\mathbf{T}^{2}\right) /$ $\operatorname{Diff}_{0}\left(\mathbf{T}^{2}\right)$ is diffeomorphic to $S L(2, \mathbb{R}) / S O(1,1)$.

Proof. Let us consider an approximately isometric system. To see the properness, we improve lemma 3.6 by noting that the linear part of the affine maps involved can be chosen in $G L(2, \mathbb{Z})$. As $\operatorname{Diff}\left(\mathbf{T}^{2}\right) / \operatorname{Diff}_{0}\left(\mathbf{T}^{2}\right)$ is isomorphic to $G L(2, \mathbb{Z})$, the diffeomorphisms of an approximately isometric system cannot stay in $\operatorname{Diff}_{0}\left(\mathbf{T}^{2}\right)$.

Let us show now that the quotient is diffeomorphic to $S L(2, \mathbb{R}) / S O(1,1)$, the space of quadratic forms of signature $(1,1)$ and of determinant 1 . Let $h$ be a flat metric. As $h$ is complete, there exists a diffeomorphism $\psi$ such that $\psi^{*} h$ is a 
quadratic form. As $\operatorname{Diff}\left(\mathbf{T}^{2}\right) / \operatorname{Diff}\left(\mathbf{T}^{2}\right)$ is isomorphic to $G L(2, \mathbb{Z})$, there exists a unique element $N$ of $G L(2, \mathbb{Z})$ such that $\psi$ is equivalent to $N$ modulo $\operatorname{Diff}_{0}\left(\mathbf{T}^{2}\right)$. Thus, $\psi \circ N^{-1}$ lies in $\operatorname{Diff}_{0}\left(\mathbf{T}^{2}\right)$ and $\left(\psi \circ N^{-1}\right)^{*} h$ is the only quadratic form in the $\operatorname{Diff}_{0}\left(\mathbf{T}^{2}\right)$-orbit of $h$.

This proposition implies that the properties of the action of $\operatorname{Diff}\left(\mathbf{T}^{2}\right)$ on $\mathcal{F}\left(\mathbf{T}^{2}\right)$ are the same as those of the left action of $\operatorname{Diff}\left(\mathbf{T}^{2}\right) / \operatorname{Diff}\left(\mathbf{T}^{2}\right)$ on the set of quadratic forms of signature $(1,1)$. We restrict ourself to the action of $\operatorname{Diff}^{+}\left(\mathbf{T}^{2}\right)$. As $\operatorname{Diff}^{+}\left(\mathbf{T}^{2}\right) / \operatorname{Diff}_{0}\left(\mathbf{T}^{2}\right)$ is isomorphic to $S L(2, \mathbb{Z})$, the point is now to understand the action of $S L(2, \mathbb{Z})$ to $S L(2, \mathbb{R}) / S O(1,1)$ and to show that it is ergodic.

The proof will be complete by noting that this action has the same dynamical properties as the right action of $S O(1,1)$ on $S L(2, \mathbb{Z}) \backslash S L(2, \mathbb{R})$. This is due to the fact that the lifts to $S L(2, \mathbb{R})$ of the invariant sets and functions of those actions are the same. This third action is well known, it is the action of the geodesic flow of the unitary tangent bundle of the modular surface $S L(2, \mathbb{Z}) \backslash \mathbb{H}^{2}$, where $\mathbb{H}^{2}$ is the hyperbolic plane. This manifold has finite volume and the action is known to be ergodic. This implies that our action is also ergodic. In particular, almost all flat orbits of $\mathcal{F}_{1}\left(\mathbf{T}^{2}\right)$ are dense in $\mathcal{F}_{1}\left(\mathbf{T}^{2}\right)$. Therefore we have proved:

Theorem 3.8. The action of $\operatorname{Diff}\left(\mathbf{T}^{2}\right)$ on $\mathcal{F}_{1}\left(\mathbf{T}^{2}\right)$ is ergodic.

It implies that, on the set of flat metrics, there are no non constant continuous function invariant by rescaling and by the action of $\operatorname{Diff}\left(\mathbf{T}^{2}\right)$.

Now, to complete the description, we may wonder which are the closed flat orbits. We can give the following proposition.

Proposition 3.9. The orbit of a flat metric for which all lightlike curves are closed is $C^{2}$-closed. Moreover an orbit is $C^{2}$-closed if and only if it is $C^{\infty}$-closed.

Proof. It is clear that non-closed orbits always contain an approximately isometric system whose shape is $\left(h, g_{n}, \varphi_{n}\right)$, i.e. with $\left(h_{n}\right)_{n \in \mathbb{N}}$ constant. We are going to see that such systems do not exist in the case above. We first give the following lemma.

Lemma 3.10. Let $H$ be a quadratic form of signature $(1,1)$ on $\mathbb{R}^{2}$ and $\left(M_{n}\right)_{n \in \mathbb{N}}$ be a non bounded sequence of automorphisms such that $\left({ }^{t} M_{n} H M_{n}\right)_{n \in \mathbb{N}}$ is convergent. Then there exists a sequence $\left(I_{n}\right)_{n \in \mathbb{N}}$ in $\operatorname{Is}(H)$ such that the sequence $\left(I_{n}^{-1} M_{n}\right)_{n \in \mathbb{N}}$ is bounded. Moreover there exists an $H$-lightlike vector $v$ such that $M_{n}^{-1} v$ tends to 0 .

Proof. The first part of this lemma is trivially deduced from lemma 2.6, and the second one is an immediate consequence of the shape of the elements of $O(1,1)$. 
We suppose that such an approximately isometric system exists. Then by lifting the system to $\mathbb{R}^{2}$, we can replace the diffeomorphisms by elements of $G L(2, \mathbb{Z})$, thanks to lemma 3.6. Set $v=(\tau, 1) \in A S\left(\varphi_{n}^{-1}\right)$ and let $H$ be a Lorentzian quadratic form and $\left(M_{n}\right)_{n \in \mathbb{N}}$ be a non bounded sequence in $G L(2, \mathbb{Z})$ such that $\left({ }^{t} M_{n} H M_{n}\right)_{n \in \mathbb{N}}$ is convergent. We set

$$
M_{n}^{-1}=\left(\begin{array}{ll}
q_{n} & p_{n} \\
r_{n} & s_{n}
\end{array}\right) .
$$

According to lemma 3.10, we have $M_{n}^{-1}=P_{n}^{-1} I_{n}^{-1}$, where $I_{n} \in \operatorname{Is}(H)$ and the sequence $\left(P_{n}\right)_{n \in \mathbb{N}}$, of elements of $G L(2, \mathbb{Z})$, is convergent. The sequence $\left(I_{n}\right)_{n \in \mathbb{N}}$ not being bounded, there exists a subsequence of $\left(M_{n}\right)_{n \in \mathbb{N}}$ such that $M_{n}^{-1}(v) \rightarrow 0$. We thus have $q_{n} \tau+p_{n} \rightarrow 0$. If $\tau \in \mathbb{Q}$ this sequence is stationary (cf. [H-W]) and so is the sequence $r_{n} \tau+s_{n}$ therefore the matrix cannot be invertible. Hence, $\tau \in \mathbb{R} \backslash \mathbb{Q}$ and $h$ has no compact lightlike geodesics and thus we have a contradiction.

The last assertion is immediate because the non $C^{2}$-closed orbits involve quadratic forms and linear maps and therefore the convergence can be supposed $C^{\infty}$.

\section{Remarks.}

1. After this proof, we might wonder if, on the torus, the approximately stable foliations of approximately isometric system always have dense leaves. This turns out to be false as shown by the example of [Mo] p. 48 .

2. A priori the statement of proposition 3.9 is not optimal. Actually, we can prove that for any flat metric $h$ with non compact lightlike curves there exists a non equicontinuous sequence of diffeomorphisms $\left(\phi_{n}\right)_{n \in \mathbb{N}}$ such that the sequence $\left(\phi_{n}^{*} h\right)_{n \in \mathbb{N}}$ is $C^{\infty}$-convergent (see [Mo] theorem IV.3). Anyway, it is not enough to prove that its orbit is not closed, we still have to compare $h$ with the limit of $\left(\phi_{n}^{*} h\right)_{n \in \mathbb{N}}$.

3. Let us give an example of a non flat orbit which is not $C^{1}$-closed. Let $A$ be the Anosov map, that is the torus diffeomorphism induced by the matrix $\left(\begin{array}{ll}2 & 1 \\ 1 & 1\end{array}\right)$. Let $g_{A}$ be an $A$-invariant Lorentzian (flat) metric on $\mathbf{T}^{2}$, and let $f$ be a non constant function of the torus. Let $X$ (resp. $Y$ ) be a non zero vector field of the contracting (resp. dilating) direction of $A$. We disturb $g_{A}$ along the lightlike contracting direction $X$ (using $Y$ ) by defining:

$$
h=g_{A}+f\left(Y^{b} \otimes Y^{b}\right),
$$

The metric is still Lorentzian. The sequence $\left(A^{n *} h\right)_{n \in \mathbb{N}}$ is $C^{1}$-convergent and its limit is $g_{A}$, but $h$ is not flat. Hence, there exist orbits which are not $C^{1}$-closed but which are $C^{2}$-closed. 


\section{The set of metrics without isometries}

If $M$ is a closed manifold, we recall that, in the Riemannian case, the set of metrics without non trivial isometries, that we will denote by $\mathcal{L}_{T}(M)$, is an open dense subset of the space of Riemannian metrics endowed with the $C^{\infty}$ topology, see [Eb]. This result is a corollary of the slice theorem of Ebin which is now clearly not true in the Lorentzian case. Anyway, adapting the proof of Ebin (see proposition IV.4.2 of $[\mathrm{Mo}])$ we still have:

Proposition 4.1. The set $\mathcal{L}_{T}(M)$ of metrics without non trivial isometries is dense in $\mathcal{L}(M)$.

The main point is to show that this set is open. Unlike to the Riemannian case the answer is different according to which manifold we consider.

\subsection{A positive result}

Thanks to corollary 3.4 and the theorem of D. Ebin, we can give the following result.

Theorem 4.2. Let $M$ be the 2-torus or a manifold such that $\operatorname{Diff}(M)$ acts properly, for the $C^{\infty}$-topology, on $\mathcal{L}(M)$. Then $\mathcal{L}_{T}(M)$ is an open (dense) set.

Proof. Let $M$ be a manifold as in the statement of the theorem. Let $\left(h_{n}\right)_{n \in \mathbb{N}}$ be a $C^{\infty}$ convergent sequence of Lorentzian metrics on $M$, such that for all $n \in \mathbb{N}$ the group of isometries of $h_{n}$, Is $\left(h_{n}\right)$, is non trivial. We are going to show that $h_{\infty}$, the limit of the sequence of metrics, also has a non trivial isometry. For this, let $\varphi_{n}$ be a non trivial element of Is $\left(h_{n}\right)$. If $\left(\varphi_{n}\right)_{n \in \mathbb{N}}$ is convergent and if its limit is not the identity map, then we have the result. If the action is proper the sequence has to be equicontinuous. If we are on the torus and if the sequence is not equicontinuous then corollary 3.4 tells us that $h_{\infty}$ is flat and therefore has a big isometry group. Consequently, in both cases, we can consider that the sequences of isometries we consider converge. Furthermore, thanks to corollary 3.4, we know that the metrics of the torus with non compact isometry groups are flat (more precisely they are invariant under an Anosov map like the metric $g_{A}$ of the remark 3. , page 478). Consequently, the isometry groups of the metrics $h_{n}$ always contain a compact subgroup. Therefore we can assume that the $\varphi_{n}$ are of finite order $p_{n}$. Now, we want to prove that there exists a sequence of integers $\left(k_{n}\right)_{n \in \mathbb{N}}$ such that $\left(\varphi_{n}^{k_{n}}\right)_{n \in \mathbb{N}}$ admits a convergent subsequence whose limit is different from the identity.

Let us suppose it is not true, then for any sequence $\left(k_{n}\right)_{n \in \mathbb{N}}$ of integers, $\varphi_{n}^{k_{n}} \rightarrow$ Id. Let $\alpha$ be a Riemannian metric with trivial isometry group. Then

\footnotetext{
1 See corollary 2.10 and corollary 3.5 .
} 
we define

$$
\alpha_{n}=1 / p_{n} \sum_{k=1}^{p_{n}}\left(\varphi_{n}^{k}\right)^{*} \alpha
$$

where $p_{n}$ is the order of $\varphi_{n}$. We have, by construction, $\varphi_{n}^{*} \alpha_{n}=\alpha_{n}$. We are going to show that $\alpha_{n} \rightarrow \alpha$, for the $C^{\infty}$-topology. For this denote by $\|\cdot\|_{l}$ the norm which gives the $C^{l}$ topology. We have:

$$
\left\|\alpha_{n}-\alpha\right\|_{l} \leq 1 / p_{n} \sum_{k=1}^{p_{n}}\left\|\varphi_{n}^{k *} \alpha_{n}-\alpha\right\|_{l} \leq m_{n},
$$

where $m_{n}=\sup _{1 \leq k \leq p_{n}}\left\|\varphi_{n}^{k *} \alpha_{n}-\alpha\right\|_{l}$. For all $n \in \mathbb{N}$, there exists an integer $k_{n}$ such that $m_{n}=\left\|\varphi_{n}^{k_{n} *} \alpha_{n}-\alpha\right\|_{l}$, therefore $m_{n} \rightarrow_{n \rightarrow \infty} 0$ and for all $l \in \mathbb{Z}$, $\left\|\alpha_{n}-\alpha\right\|_{l} \rightarrow_{n \rightarrow \infty} 0$ hence $\alpha_{n} \rightarrow_{n \rightarrow \infty} \alpha$, in a $C^{\infty}$ way. According to the work of D. Ebin, for all $n$ sufficiently large, the isometry group of $\alpha_{n}$ is conjugated to a subgroup of the isometry group of $\alpha$. That is a contradiction. Consequently $h_{\infty}$ has at least one non trivial isometry.

\section{Remarks.}

1. This result is still true for manifolds on which the limits of approximately isometric systems (the metrics $h_{\infty}$ and $g_{\infty}$ ) always possess isometries. Of course the only known example is $\mathbf{T}^{2}$.

2. It is possible to show (cf. [Mo] examples p. 48-49) that, on the torus, the result is not valid for the $C^{0}$ topology or that we can have a diminution of the number of isometries for a $C^{\infty}$-convergent sequence of metrics. But we found more interesting to show what happens to this set on the compact quotients of $P S L(2, \mathbb{R})$.

\subsection{On compact quotients of $P S L(2, \mathbb{R})$}

The goal of this section is to construct a sequence of metrics on a compact manifold with non trivial isometry groups which converges $\left(C^{\infty}\right)$ to a metric with trivial isometry group. We are going to choose the metrics in the set of left-invariant metrics of $P S L(2, \mathbb{R})$ and look at the situation on a compact quotient. Consequently, we have to determine the isometry groups of left invariant metrics on $\operatorname{PSL}(2, \mathbb{R})$. The isometry groups of left invariant Riemannian metrics on compact simple groups are well known since the works of T. Ochiai and T. Takahashi (cf. $[\mathrm{O}-\mathrm{T}]$ ) and the works of J. E. D'Atri and W. Ziller (cf. [D'A-Z] theorem 5, page 24$)$. Adapting some of their results to $P S L(2, \mathbb{R})$, we compute those groups, and thus the sequence of metrics will appear quite naturally. 


\subsubsection{Isometry groups of left invariant metrics of $P S L(2, \mathbb{R})$}

During this paragraph, we will denote by $G_{0}$ the connected component of the identity of a group $G$. We first prove a $P S L(2, \mathbb{R})$ version of the theorem of D'Atri and Ziller.

Proposition 4.3. Let $h$ be a left invariant, non biinvariant, pseudo-Riemannian metric on $G=P S L(2, \mathbb{R})$. Then the isometry group of $h$ is generated by the left translations and a subgroup of the right translations.

Proof. Let Is $(h)$ be the isometry group of $h$ and $L(G)$ the subgroup of Is $(h)$ composed of the left translations.

We first study the connected component of the identity of $\operatorname{Is}(h)$ that we denote by $\operatorname{Is}_{0}(h)$. We denote by $\operatorname{Int}(G)$ the group of inner automorphisms of $G$. We are going to use the following result:

Theorem 4.4 Ochiai and Takahashi (cf. [O-T] theorem 3). Let $G$ be a semisimple Lie group endowed with a left invariant pseudo-Riemannian metric $h$. Then $\mathrm{Is}_{0}(h) \subset L(G) \operatorname{Int}(G)$ if and only if $L(G)$ is a normal subgroup of $\operatorname{Is}_{0}(h)$.

We are going to show that $L(G)$ is a normal subgroup of $\operatorname{Is}_{0}(h)$. Let us give an upper bound for the dimension of $\operatorname{Is}(h)$. It is well known that the dimension of the isometry group of an $n$ dimensional pseudo-Riemannian manifold is at most $n(n+1) / 2$. Moreover we know that in this case the sectional curvature is constant. In our case it means that the metric is biinvariant. So, here, the dimension is at most 5 . Moreover, if the isometry group contains a 5 dimensional subgroup it also implies that the curvature is constant and the metric is biinvariant (it can be proved directly from a study of the action of the isometry group on the 2-Grassmannian but it can also be deduced from the systematic study of the isometry groups of Lorentz 3-manifolds done by C. Bona et B. Coll in [B-C]). Therefore we have dim $\operatorname{Is}_{0}(h)=3$ or 4 . We denote by $\mathfrak{I}$ the Lie algebra of $\operatorname{Is}_{0}(h)$ and $\mathfrak{g}$ the subalgebra of $\mathfrak{I}$ corresponding to $L(G)$. We want to show that $\mathfrak{g}$ has to be an ideal of $\mathfrak{I}$. If $\operatorname{dim} \mathfrak{I}=3$ it is obvious. Let us study the case where $\operatorname{dim} \mathfrak{I}=4$. The Lie algebra cannot be semisimple. Thus its radical, $\operatorname{rad}(\mathfrak{I})$, is not trivial and its dimension is at most 1 because it cannot intersect $\mathfrak{g}$ which is simple. Let us take two elements $x$ and $y$ of $\mathfrak{g}$ and $u$ a non zero element of $\operatorname{rad}(\mathfrak{I})$. According to Jacobi identity we have

$$
[[x, y], u]+[[u, x], y]+[[y, u], x]=0 .
$$

However, $\operatorname{since} \operatorname{rad}(\mathfrak{I})$ is a one dimensional ideal, this implies

$$
[[u, x], y]+[[y, u], x]=0,
$$

and therefore $[[x, y], u]=0$. As $[\mathfrak{g}, \mathfrak{g}]=\mathfrak{g}$, we find that $\mathfrak{g}$ is an ideal of $\mathfrak{I}$ therefore $\mathrm{Is}_{0}(h)$ can be written $L(G) \times K$, with $K$ a one dimensional subgroup of the group of right translations whose Lie algebra is $\operatorname{rad}(\mathfrak{I})$. 
To finish the proof, we use the following theorem of J. E. D'Atri and W. Ziller. It is not exactly the same statement as the original version (compare with theorem 5 page 24 of [D'A-Z]) but the proof is exactly the same.

Theorem 4.5 (D'Atri and Ziller (cf. [D'A-Z])). Let $G$ be a simple Lie group on which any left invariant pseudo-Riemannian metric $h$ satisfies $\operatorname{Is}_{0}(h) \subset L(G) \operatorname{Int}(G)$. Let $\varphi$ be an isometry between two non biinvariant left invariant metrics $h$ and $h^{\prime}$. Then $\varphi$ is an automorphism of $G$.

This theorem implies that $\operatorname{Is}(h) \subset \operatorname{Aut}(G)$. To conclude we note that

$$
\operatorname{Int}(S L(2, \mathbb{R}))=\operatorname{Aut}(S L(2, \mathbb{R}))
$$

(cf. [O-V] for example).

Remark. Thanks to this result finding the isometry group of a left invariant metric is now a problem of linear algebra. More precisely, we are going to see that finding the subgroup $K$ is the same as finding the intersection between the isometry groups of two quadratic forms of $\mathbb{R}^{3}$. Actually, we just want the set of isometries that fix the neutral element of $\operatorname{PSL}(2, \mathbb{R})$. For a metric $h$ we will denote it by Isot $(h, e)$. Proposition 4.3 entails that if $h$ is a left invariant metric then $\operatorname{Isot}(h, e)$ is contained in $\operatorname{Int}(P S L(2, \mathbb{R}))$. For the Killing metric Isot $(K i l l i n g, e)$ is the whole of $O(2,1)$ (see $[\mathrm{Sa}]$, proposition 2.3.2.5, for a nice geometric study of the isometry group of the Killing form), moreover $\operatorname{Isotr}_{0}($ Killing,e) (the connected component of the identity) is the whole group of inner automorphisms. Therefore in order to know $\operatorname{Is}(h)$ it is sufficient to compute $S O_{0}(2,1) \cap \operatorname{Is}\left(h_{e}\right)$, where $\operatorname{Is}\left(h_{e}\right)$ denotes the group of linear isometries of the quadratic form obtained by restricting $h$ to $T_{e} G$.

If we choose a left invariant trivialisation of the tangent bundle, we can represent the Killing form by a symmetric matrix $K$ and a left invariant metric $h$ by a matrix $H$. Hence we can associate to $h$ the endomorphism, $N_{h}$, of $\mathbb{R}^{3}$ whose matrix is $H K^{-1}$. It is well known that both quadratic forms are simultaneously diagonalizable if and only if $N_{h}$ is diagonalizable.

From this discussion, and some classical computations, we can deduce the following proposition (see $[\mathrm{Mo}]$ for the proof and a more detailed version).

Proposition 4.6. Let $h$ be a left invariant Lorentzian metric on $P S L(2, \mathbb{R})$.

1. If all the eigenvalues of $N_{h}$ are distinct then Is $(h)$ is isomorphic to $\operatorname{PSL}(2, \mathbb{R}) \times \mathbb{Z}_{2}$.

2. If $N_{h}$ is not diagonalizable and has two distinct eigenvalues or has only one eigenvalue and a 1-dimensional eigenspace then $\operatorname{Is}(h)$ is isomorphic to $\operatorname{PSL}(2, \mathbb{R})$.

The metrics corresponding to the second case possess a left invariant lightlike geodesic foliation of codimension 1 whose tangent is the lightlike plane that the 
metric and the Killing form have in common. It is not hard to see (thanks to the precious proposition 3.18 of [C-E]), that left-invariant metrics which possess such lightlike 2-foliations are those which have a lightlike plane in common with the Killing form.

\subsubsection{On the compact quotients}

On some compact manifolds, we want to construct a sequence of metrics $\left(\bar{h}_{n}\right)_{n \in \mathbb{N}}$ with isometry group $\mathbb{Z}_{2}$ which tends to a metric $\bar{h}_{\infty}$ with trivial isometry group. Of course, as we have seen in the proof of theorem $4.2, \bar{h}_{\infty}$ will possess a lightlike geodesic foliation of codimension 1 . This is quite similar to the situation described in proposition 4.6. Thus, we try to find our example on $\operatorname{PSL}(2, \mathbb{R})$ among left invariant metrics.

We choose a left invariant trivialisation of the tangent bundle of $\operatorname{PSL}(2, \mathbb{R})$ such that the matrix of the Killing form in it is:

$$
\left(\begin{array}{lll}
0 & 1 & 0 \\
1 & 0 & 0 \\
0 & 0 & 1
\end{array}\right) .
$$

For all $n>1$, we define $h_{n}$ as the left invariant metric whose matrix in this trivialisation is

$$
\left(\begin{array}{ccc}
0 & \alpha & 1 / n \\
\alpha & 0 & \gamma \\
1 / n & \gamma & \delta
\end{array}\right)
$$

with $\alpha, \gamma$ et $\delta$ in $\mathbb{R}_{+}^{*}$. It is easy to see that, for all $n$, the matrix $N_{h_{n}}$ has three distinct eigenvalues. Hence, according to proposition 4.6, their isometry groups are all isomorphic to $P S L(2, \mathbb{R}) \times \mathbb{Z} / 2 \mathbb{Z}$. The sequence of quadratic forms defined by those matrices converges, therefore the sequence of left invariant metrics converges $C^{\infty}$. We denote the limit by $h_{\infty}$. The matrix associated to $h_{\infty}$ is clearly:

$$
\left(\begin{array}{lll}
0 & \alpha & 0 \\
\alpha & 0 & \gamma \\
0 & \gamma & \delta
\end{array}\right) .
$$

We notice that $N_{h_{\infty}}$ is not diagonalizable and has two different eigenvalues, therefore, according to proposition 4.6 , the isometry group of $h_{\infty}$ is reduced to the left translations. Hence, we have already lost an isometry. Let us take the quotient by a cocompact lattice of $P S L(2, \mathbb{R})$ and see what happens.

Let $\Gamma$ be a cocompact fuschian subgroup of $\operatorname{PSL}(2, \mathbb{R})$. We denote by $V$ the quotient manifold $\Gamma \backslash \mathrm{SL}(2, \mathbb{R})$, by $\Sigma$ the compact hyperbolic surface $\Gamma \backslash \mathbb{H}^{2}$ endowed with its natural Riemannian metric, by $N(\Gamma)$ the normalizer of $\Gamma$ in $\operatorname{PSL}(2, \mathbb{R})$ and by $\bar{h}$ the metric induced on $V$ by a left invariant metric $h$ of $\operatorname{PSL}(2, \mathbb{R})$. Clearly if $\operatorname{Is}(h)=S L(2, \mathbb{R}) \times K$, we have $\operatorname{Is}(\bar{h})=N(\Gamma) / \Gamma \times K$. But we have a geometrical interpretation of $N(\Gamma) / \Gamma$, it is the isometry group of $\Sigma$. However, in genus greater than 2, there exist (and it is the generic case) hyperbolic surfaces 
with trivial isometry group (see for example [Bu] in the proof of theorem 6.5.3). If $\Gamma^{\prime}$ is a group corresponding to such a surface it satisfies:

$$
N\left(\Gamma^{\prime}\right) / \Gamma^{\prime}=\{e\} .
$$

Consequently, since in the case of $h_{\infty}$ we have $K=\{\operatorname{Id}\}$, we have shown that

$$
\operatorname{Is}\left(\bar{h}_{\infty}\right)=\{I d\} .
$$

The following statement is therefore proved.

Theorem 4.7. There exist compact manifolds $V$, of dimension greater than 2 such that the set of metrics without isometries is not an open subset of $\mathcal{L}(V)$ endowed with the $C^{\infty}$ topology.

Remark. Thanks to the article [G-L] of M. Guediri and J. Lafontaine, the same sequence of metrics enables us to see that the set of complete metrics is not closed in general (it is, of course, not open). It is not a surprise. Indeed, as we saw in lemma 2.4, the result of [R-S] on the completeness of a geodesic obtained as a limit can be adapted to the completeness of a metric obtained as a limit.

\section{References}

[Ad] J. F. Adams, Vector fields on sphere, Annals of Math. (2) 75 (1962), 603-632.

[A-S] S. Adams and G. Stuck, The isometry group of a compact Lorentz manifold. I, II, Invent. Math. 129 (1997), 239-261, 263-287.

[B-N] G. Birman and K. Nomizu, The Gauss-Bonnet theorem for 2-dimensional spacetimes, Michigan Math. J. 31 (1984), 77-81.

[B-C] C. Bona and B. Coll, Isometry groups of three-dimensional Lorentzian metrics, J. Math. Phys. 35 (1994) no. 2, 873-884.

[Bu] P. Buser, Geometry and spectra of compact Riemann surfaces, Progress in Mathematics 106, Birkhäuser Boston, Inc., Boston, MA, 1992.

[C-R] Y. Carrière et L. Rozoy, Complétude des métriques lorentziennes de $T^{2}$ et difféomorphismes du cercle, Bol. Soc. Brasil. Mat. (N.S.) 25 (1994), no. 2, 223-235.

[C-E] J. Cheeger and D. G. Ebin, Comparison theorems in Riemannian geometry, NorthHolland Mathematical Library, Vol. 9, North-Holland Publishing Co., AmsterdamOxford; American Elsevier Publishing Co., Inc., New York, 1975.

[D-Gr] G. D'Ambra and M. Gromov, Lectures on transformation groups: geometry and dynamics, Surveys in Differential Geometry (supplement to the Journal of Differential Geometry) 1 (1991), 19-111.

[D'A-Z]J. E. D'Atri and W. Ziller, Naturally reductive metrics and Einstein metrics on compact Lie groups, Mem. Amer. Math. Soc. 18 (1979), no. 215.

[Eb] D.G. Ebin, The manifold of Riemannian metrics, 1970 Global Analysis (Proc. Sympos. Pure Math., Vol. XV, Berkeley, Calif., 1968) 11-40, Amer. Math. Soc., Providence, R.I.

[G-L] M. Guediri et J. Lafontaine, Sur la complétude des variétés pseudo-riemanniennes, J. Geom. Phys. 15 (1995), no. 2, 150-158.

[H-W] G. H. Hardy and E. M. Wright, An introduction to the theory of numbers, The Clarendon Press, Oxford University Press, New York, 1979.

$[\mathrm{H}-\mathrm{H}]$ G. Hector and U. Hirsch, Introduction to the geometry of foliations. Part A, Aspects of Mathematics, 1. Friedr. Vieweg \& Sohn, Braunschweig, 1986. 
[Mo] P. Mounoud, Sur l'espace des métriques de Lorentz d'une variété compacte, Thèse de l'Université Montpellier II, 2001.

[O-T] T. Ochiai and T. Takahashi, The group of isometries of a left invariant Riemannian metric on a Lie group, Math. Ann. 223 (1976), no. 1, 91-96.

$[\mathrm{O}-\mathrm{V}]$ A. L. Onishchik and É. B. Vinberg, Lie groups and algebraic groups, Springer Series in Soviet Mathematics, Springer-Verlag, Berlin, 1990.

[R-S] S. Romero and M. Sanchez, On the completeness of geodesics obtained as a limit, J. Math. Phys. 34 (8), August (1993), 3769-3774.

[Sa] F. Salein, Variétés anti De Sitter de dimension 3, Thèse de l'ENS Lyon, 1999.

[Ze1] A. Zeghib, Sur les espaces-temps homogènes, Geometry and Topology Monographs 1 (1998), 531-556.

[Ze2] A. Zeghib, The identity component of the isometry group of a compact Lorentz manifold, Duke Math. J. 92 (1998), 321-333.

[Ze3] A. Zeghib, Geodesic foliations in Lorentz 3-manifolds, Comment. Math. Helv. 74 (1999), no. $1,1-21$.

[Ze4] A. Zeghib, Isometry groups and geodesic foliations of Lorentz manifolds. I. Foundations of Lorentz dynamics, Geom. Funct. Anal. 9 (1999), no. 4, 775-822.

[Zi] R. Zimmer, On the automorphism group of a compact Lorentz manifold and other geometric manifolds, Invent. Math. 83 (1986), no. 3, 411-424.

Pierre Mounoud

Département de Mathématiques

GTA (CNRS UMR 5030)

Université Montpellier II

case 51

34095 Montpellier

France

e-mail: mounoud@math.univ-montp2.fr

(Received: November 2, 2001)

To access this journal online:

(I0) http://www.birkhauser.ch 